\title{
EVALUATION OF BROOMRAPE RESISTANCE IN SUNFLOWER HYBRIDS
}

\author{
Kaya, Y.*, Evci, G., Pekcan, V., Gucer T., Yilmaz, M.I.
}

Trakya Agricultural Research Institute, P.O.Box: 16, 22100 Edirne, Turkey

Received: July 15, 2009

Accepted: November 10, 2009

\begin{abstract}
SUMMARY
Broomrape (Orobanche cernua Loeffl.) is the most serious problem in sunflower production in Turkey, some East European countries and Spain. A research covering broomrape tests of sunflower hybrids in the trials based on National Sunflower Research Project was conducted in Trakya region, which holds 75\% of the sunflower production in Turkey, in 2006, 2007 and 2008. Based on this project, in conditions of natural infestation, 306, 443 and 533 commercial and candidate hybrids, inbred lines, etc. were tested in 2006, 2007 and 2008, respectively. Of the tested materials, 12 in 2006, 56 in 2007 and 140 in 2008 were found to be resistant. During winter, broomrape tests continued in growth chambers and in pots, under conditions of artificial infestation. Broomrape seeds were collected from a naturally infested area in Trakya region and used to determine the reaction of developed hybrids and lines to the collected races. In this part of the study, 1752, 816 and 1389 genetic material were tested in 2006, 2007 and 2008, respectively. The tests showed that 332, 94, and 404 materials in 2006, 2007 and 2008, respectively, were resistant to the new races of broomrape used in the study.
\end{abstract}

Key words: sunflower, broomrape, tests, resistance

\section{INTRODUCTION}

Broomrape (Orobanche cernua Loeffl.) has been the most serious problem in sunflower production in Turkey, leading to considerable yield losses, up to $100 \%$, and reducing of sunflower seed quality since 1960s. Furthermore, this parasite develops new and more virulent races year after year which overcome the resistance of the varieties and hybrids used in commercial production. Broomrape flowers produce a large number of very small seeds which fall to soil surface and so the parasite spreads easily and quickly by wind and it cannot be controlled efficiently with cultural methods such as rotation, later planting, etc.

* Corresponding author: Phone: +90 284 2358182; Fax: +90 284 2358210; e-mail: yalcinkaya@ttae.gov.tr 
Although old races are known to exists in many countries (Sukno et al, 1999; Tang et al., 2003; Shindrova, 2006), new races of Orobanche occurred only in Spain (F-Martínez et al. 2000; Melero-Vara et al., 2000; Aktouch et al., 2002; Pérez-Vich et al., 2002) and Turkey (Kaya et al., 2004b). However, the virulence of these new races was overcome by resistant hybrids (Jan et al., 2002) and imidazilonone herbicides applyed post emergence in the so-called CLEARFIED ${ }^{\circledR}$ system (Kaya et al., 2004a).

\section{MATERIAL AND METHODS}

Sunflower hybrids and lines were developed within National Sunflower Research Project at TARI (Trakya Agricultural Research Institute) in Edirne and the commercial sunflower hybrids that belong to other companies and institutions were tested against the new broomrape races under natural and artificial conditions in the period 2006-2008.

Under the conditions of artificial infestation, broomrape seeds were cleaned threshing the plants collected from different infested fields of Trakya Region having $75 \%$ of Turkey sunflower production. Broomrape tests were performed in pots, mainly in winter time. Before planting, about 1-2 g broomrape seeds were placed at the bottom of the pots. Orobanche plants were counted on each root after 35 days from planting, after washing the rots.

Under the natural conditions, broomrape test were conducted in infested Malkara region each year. Susceptible Sanbro hybrid and resistant P-4223 hybrid were used as controls at each 40 rows which were $1 \mathrm{~m}$ long between rows at $4 \mathrm{~m}$ plots. Broomrapes were counted on each plant in the rows between flowering and physiological stages. Broomrape occurrence was evaluated for frequency (F), intensity (I) and attacking rate (AR) based on Pustovoit method. Plants were accepted as resistant when having 0-10 frequency \%, and 0-1 AR values (Vranceanu et al., 1980). The plants with 10-20 frequency \% were accepted as tolerant.

- $\mathrm{F}=\%$ of plants with Orobanche (the plant number infested by Orobanche/total number of plants in the row $\times 100$ )

- I=the number of Orobanche in one infested plant (total Orobanche/total plants infested by Orobanche in the row)

- $\mathrm{AR}=\mathrm{F} \times \mathrm{I} / 100=$ the number of Orobanche on one plant in the row.

\section{RESULTS AND DISCUSSION}

In the conditions of natural infestation, 1282 genetic material were tested between 2006 and 2008. Resistant materials were increased extremely (from 12 to 140 ) in last year (Table 1). Similarly, the number of resistant material were $50 \%$ more than 2006s observations (Table 2). These results indicated that sunflower breeding program at TARI improved efficiently broomrape resistance. Materials that belonged to institutions other than TARI were also tested in this period. Although there were no resistant lines from Dobroudja, Szeged and Karaj institutes, 
Uludag University and USDA Sunflower Research Unit, some resistant lines and hybrids were observed in the material from Soltis and Fundulea Institute (Tables 3, 4 and 5). From the sunflower hybrids which are commonly sold on the Turkish market, only P-4223 from Pioneer Seed Co, Tunca from Limagrain Seed Co and Muson from May Seed Co. showed resistance in this study (Tables 6, 7 and 8).

Table 1: Broomrape tests under the conditions of natural infestation conducted in summer

\begin{tabular}{lccc}
\hline \multirow{2}{*}{ Type } & \multicolumn{3}{c}{ Year } \\
\cline { 2 - 4 } & 2006 & 2007 & 2008 \\
\hline Number of tested material & 306 & 443 & 533 \\
Number of resistant material & 12 & 56 & 140 \\
\hline
\end{tabular}

Table 2: Broomrape tests in pots conducted in winter time in the period 2006-2008

\begin{tabular}{lcccc}
\hline Year & Tested material & Susceptible & Tolerant (1-5 Bromrape) & Resistant \\
\hline 2006 & 1702 & 1072 & 278 & 352 \\
2007 & 2167 & 1501 & 389 & 277 \\
2008 & 1389 & 721 & 264 & 404 \\
\hline
\end{tabular}

Table 3: Broomrape tests under conditions of natural infestation conducted in Malkara in 2006

\begin{tabular}{lccccc}
\hline Institution & Type & Total material & Susceptible & Tolerant & Resistant \\
\hline Dobroudja-Bulgaria & cms Line & 13 & 13 & - & - \\
Szeged GKI-Hungary & cms Line & 10 & 10 & - & - \\
TARI & Candidate Hybrid & 40 & 38 & 1 & 1 \\
TARI $\times$ Maisadour & Joint Hybrid & 9 & 9 & - & - \\
TARI $\times$ Szeged & Joint Hybrid & 33 & 33 & - & - \\
TARI $\times$ Soltis & Joint Hybrid & 72 & 66 & 5 & 1 \\
TARI $\times$ Fundulea & Joint Hybrid & 6 & 6 & - & - \\
TARI $\times$ Novi Sad & Joint Hybrid & 12 & 12 & - & - \\
Soltis-France & Hybrid & 40 & 33 & 4 & 3 \\
Karaj-Iran & Hybrid & 6 & 6 & - & - \\
FAO Network Trials & Hybrid & 13 & 12 & 1 & - \\
Registration Trials (Oil Type) & Hybrid & 23 & 17 & 2 & 4 \\
\hline
\end{tabular}

Table 4: Broomrape tests under conditions of natural infestation conducted in Malkara in 2007

\begin{tabular}{llcccc}
\hline Institution & Type & Total material & Susceptible & Tolerant & Resistant \\
\hline Soltis-France & cms Line & 10 & 4 & 3 & 3 \\
Szeged GKI-Hungary & cms Line & 5 & 5 & - & - \\
Fundulea Romania & cms Line & 7 & - & 2 & 5 \\
USDA Germplasm & cms Line & 19 & 18 & 1 & - \\
USDA Germplasm & Restorer Line & 11 & 9 & 2 & - \\
TARI & Candidate Hybrid & 47 & 6 & 3 & 37 \\
Hibrisol-Spain & Candidate Hybrid & 41 & 27 & 11 & 3 \\
Uludag University & Candidate Hybrid & 6 & 5 & 1 & - \\
TARI $\times$ Dobroudja & Joint Hybrid & 62 & 60 & 2 & - \\
TTAE $\times$ Szeged GKI & Joint Hybrid & 119 & 105 & 12 & 2 \\
TTAE $\times$ Maisadour & Joint Hybrid & 18 & 17 & 1 & - \\
Registration Trials (oil type) & Hybrids & 33 & 22 & 4 & 7 \\
\hline
\end{tabular}


Table 5: Broomrape tests under conditions of natural infestation conducted in Malkara in 2008

\begin{tabular}{llcccc}
\hline Institution & Type & $\begin{array}{c}\text { Total } \\
\text { material }\end{array}$ & Susceptible & Tolerant & Resistant \\
\hline TARI & Cms Line & 18 & - & - & 18 \\
TARI & Candidate Hybrid & 98 & 60 & 2 & 36 \\
AARI & Candidate Hybrid & 12 & 11 & - & 1 \\
TARI $\times$ Szeged GKI & Joint Hybrid & 134 & 100 & 2 & 32 \\
TARI $\times$ Soltis & Joint Hybrid & 26 & 16 & 1 & 9 \\
TARI $\times$ Hibrisol & Joint Hybrid & 7 & 2 & - & 5 \\
TARI $\times$ Maisadour & Joint Hybrid & 14 & 6 & 1 & 7 \\
TARI $\times$ Novi Sad & Joint Hybrid & 10 & 5 & - & 5 \\
TARI $\times$ Fundulea & Joint Hybrid & 10 & 10 & - & - \\
AGROMAR & Hybrid & 3 & 2 & - & 1 \\
Registration Trials (Oil Type) & Hybrid & 22 & 2 & - & 20 \\
Registration Trials (Confectionery) & Hybrid & 6 & 6 & - & - \\
\hline
\end{tabular}

Table 6: Broomrape resistance of the most popular commercial hybrids in Turkey tested in Malkara in 2006

\begin{tabular}{lcccccc}
\hline Cultivar & Plant \# & $\begin{array}{c}\text { Plant \# with } \\
\text { Orobanche }\end{array}$ & $\begin{array}{c}\text { Orobanche } \\
\text { number }\end{array}$ & $\begin{array}{c}\text { Frequency } \\
(\%)\end{array}$ & Intensity & $\begin{array}{c}\text { Attacking } \\
\text { rate }\end{array}$ \\
\hline P-4313 & 10 & 9 & 50 & 90 & 5.55 & 5.00 \\
P-4223 & 10 & 0 & 0 & 0 & 0 & 0 \\
MUSON & 10 & 0 & 0 & 0 & 0 & 0 \\
MERIC & 10 & 9 & 55 & 90 & 6.11 & 5.50 \\
PACTOL & 10 & 9 & 50 & 90 & 5.55 & 5.00 \\
SANBRO & 10 & 9 & 60 & 90 & 6.66 & 6.00 \\
COBAN & 10 & 9 & 57 & 90 & 6.33 & 5.70 \\
C-70165 & 10 & 9 & 53 & 90 & 5.88 & 5.30 \\
TUNCA & 10 & 0 & 0 & 0 & 0 & 0 \\
\hline
\end{tabular}

Table 7: Broomrape resistance of the most popular commercial hybrids in Turkey tested in Malkara in 2007

\begin{tabular}{lcccccc}
\hline Cultivar name & Plant \# & $\begin{array}{c}\text { Plant \# with } \\
\text { Orobanche }\end{array}$ & $\begin{array}{c}\text { Orobanche } \\
\text { number }\end{array}$ & $\begin{array}{c}\text { Frequency } \\
(\%)\end{array}$ & Intensity & $\begin{array}{c}\text { Attacking } \\
\text { rate }\end{array}$ \\
\hline C-70165 & 7 & 4 & 7 & 57 & 1.75 & 1.00 \\
COBAN & 7 & 4 & 11 & 57 & 2,75 & 1.57 \\
MERIC & 10 & 2 & 3 & 20 & 1.50 & 0.30 \\
DKF-2525 & 10 & 1 & 1 & 10 & 1.00 & 0.10 \\
MUSON & 8 & 0 & 0 & 0 & 0 & 0 \\
P-4223 & 8 & 0 & 0 & 0 & 0 & 0 \\
SANBRO & 8 & 3 & 7 & 38 & 2.33 & 0.88 \\
TUNCA & 7 & 0 & 0 & 0 & 0 & 0 \\
\hline
\end{tabular}

Table 8: Broomrape resistance of the most popular commercial hybrids in Turkey tested in Malkara in 2008

\begin{tabular}{lcccccc}
\hline Cultivar & Plant \# & $\begin{array}{c}\text { Plant \# with } \\
\text { Orobanche }\end{array}$ & $\begin{array}{c}\text { Orobanche } \\
\text { number }\end{array}$ & $\begin{array}{c}\text { Frequency } \\
(\%)\end{array}$ & $\begin{array}{c}\text { Intensity } \\
\text { Attacking } \\
\text { rate }\end{array}$ \\
\hline MERIC & 5 & 3 & 10 & 60 & 3.33 & 2.00 \\
P-4223 & 5 & 0 & 0 & 0 & 0 & 0 \\
DKF-2525 & 5 & 1 & 1 & 20 & 1.00 & 0.20 \\
SANBRO & 6 & 4 & 8 & 66 & 2.00 & 1.32 \\
TUNCA & 6 & 0 & 0 & 0 & 0 & 0 \\
\hline
\end{tabular}


Each year, resistant sunflower hybrids from different seed companies are released on the market. DKF-2525, from Monsanto Seed Co., released in 2006, was observed together with other resistant commercial hybrids and some broomrape plants were observed on its roots (Tables 7 and 8). While only some materials were observed as resistant in 2006 (Table 9), many lines and hybrids exhibited resistant behavior in 2007 and 2008 (Tables 10 and 11).

Table 9: Broomrape tests under conditions of natural infestation conducted in Malkara in 2006

\begin{tabular}{lllccc}
\hline$\#$ & Material & Origin & Type & Plant \# & $\begin{array}{c}\text { Plant \# with Orobanche } \\
\text { Orobanche }\end{array}$ \\
& number \\
\hline 1 & Tunca & Sygenta Commercial Hybrid & 10 & 1 & 1 \\
3 & Muson & Sygenta Commercial Hybrid & 10 & 1 & 1 \\
5 & $2478-A$ & TARI cms Line & 10 & 1 & 1 \\
$C$ & P-4223 (Resistant Check) & Pioneer Commercial Hybrid & 10 & 0 & 0 \\
C & SANBRO (Susceptible Check) & Sygenta Commercial Hybrid & 10 & 9 & $>50$ \\
70 & ED/9 & SOLTIS Hybrid & 10 & 0 & 0 \\
86 & ED/25 & SOLTIS Hybrid & 10 & 0 & 0 \\
95 & ED/34 & SOLTIS Hybrid & 10 & 0 & 0 \\
106 & SOLF 2585j/A & SOLTIS cms Line & 10 & 0 & 0 \\
164 & LG1234/A $\times$ 6973-R & SOLTIS Joint Hybrid & 0 & 0 & 0 \\
203 & 2517-A $\times 9216-R$ & TARI $\quad$ Candidate Hybrid & 10 & 0 & 0 \\
\hline
\end{tabular}

Both joint hybrids with other institutions and the hybrids from TARI were observed in the study, especially in 2007 and 2008 (Tables 10 and 11). Additionally, both finished inbred lines from TARI, early breeding lines and numerous $\mathrm{F}_{4} \mathrm{~F}_{5}$ and $\mathrm{F}_{6}$, genetic material were found resistant both under natural and artificial conditions in the winter tests. The increased number of resistant material lines and hybrids, especially in recent years, showes the success of the sunflower breeding program conducted at TARI.

Results on broomrape resistance results for some years were not entirely reliable due to drought during sunflower growing season and inconsistency in broomrape distribution in all parts of the experiment field. Therefore, to confirm the results of natural infestation and also previous control of genetic material before planting in breeding nursery, the material evaluated under natural conditions was also tested against new broomrape races under conditions of artificial infestation.

\section{CONCLUSIONS}

There was considerable variation in broomrape resistance among the inbred lines and hybrids screened in this study. Resistant inbred lines and hybrids from TARI and other institutions were observed both in natural and artificial conditions because the final objective is to develop material resistant to new broomrape races for Turkish market (about 2.000 MT hybrid seed). There is no option to sell nonre- 
Table 10: Broomrape tests under conditions of natural infestation conducted in Malkara in 2007

\begin{tabular}{|c|c|c|c|c|c|}
\hline$\overline{\#}$ & Material & Origin & Type & Total plant & Result \\
\hline 1 & F5AA410/A & Soltis & cms Line & 10 & $\bar{R}$ \\
\hline 5 & SF49CC01JO/A XEGF646J-0 & Soltis & cms Line & 10 & $\mathrm{R}$ \\
\hline 10 & SOLF0618 J/A $\times$ SP-R-96 & Soltis & cms Line & 10 & $\mathrm{R}$ \\
\hline C & P-4223 (Resistant Check) & Pioneer & Commercial Hybrid & 10 & $\mathrm{R}$ \\
\hline C & SANBRO (Susceptible Check) & Sygenta & Commercial Hybrid & 10 & S \\
\hline 118 & 3009-A × K4-R S.N:53 & TARI & Candidate Hybrid & 10 & $\mathrm{R}$ \\
\hline 119 & 7751-A × K6-R S.N:10-15 & TARI & Candidate Hybrid & 10 & $R$ \\
\hline 120 & HA-89-1-A × K7-R S.N:6 & TARI & Candidate Hybrid & 10 & $\mathrm{R}$ \\
\hline 121 & 0195-A × MMR-471-2 S.N:8 (2006) & TARI & Candidate Hybrid & 10 & $\mathrm{R}$ \\
\hline 122 & HA-821-A × MMR-471-2 S.N:8 (2006) & TARI & Candidate Hybrid & 10 & $\mathrm{R}$ \\
\hline 123 & 2517-A $\times$ MMR-471-2 S.N:6 (2006) & TARI & Candidate Hybrid & 10 & $\mathrm{R}$ \\
\hline 124 & $2517-A \times$ K3-R S.N:14,15,16 & TARI & Candidate Hybrid & 10 & $\mathrm{R}$ \\
\hline 126 & 2517-A × K3-R S.N:33,34,35,36 & TARI & Candidate Hybrid & 10 & $R$ \\
\hline 127 & 2453-A × K3-R S.N:17,18,19 & TARI & Candidate Hybrid & 10 & $\mathrm{R}$ \\
\hline 225 & $\mathrm{H}-114-\mathrm{H}-115-\mathrm{HO} \times \mathrm{K} 7-\mathrm{R} \mathrm{SN}: 6$ & TARI & Candidate Hybrid & 10 & $\mathrm{R}$ \\
\hline 231 & 6626-A × K4-R S.N:53 & TARI & Candidate Hybrid & 10 & $\mathrm{R}$ \\
\hline 232 & 62001-A × K4-R S.N:46 & TARI & Candidate Hybrid & 10 & $\mathrm{R}$ \\
\hline 233 & 62003-A × K4-R S.N:46 & TARI & Candidate Hybrid & 10 & $\mathrm{R}$ \\
\hline 234 & 6522-A × K4-R S.N:51,52 & TARI & Candidate Hybrid & 10 & $\mathrm{R}$ \\
\hline 235 & 6522-A × K4-R S.N:53 & TARI & Candidate Hybrid & 10 & $\mathrm{R}$ \\
\hline 236 & 65371-A × MMR-471-2 S.N:8 (2006) & TARI & Candidate Hybrid & 10 & $\mathrm{R}$ \\
\hline 237 & 3009-A × K7-R SN:6 & TARI & Candidate Hybrid & 10 & $\mathrm{R}$ \\
\hline 239 & 7751-A × K7-R SN:6 & TARI & Candidate Hybrid & 10 & $\mathrm{R}$ \\
\hline 240 & 62003-A × K7-R SN:6 & TARI & Candidate Hybrid & 10 & $R$ \\
\hline 241 & $6398-A \times K 6-R$ SN:10-15 & TARI & Candidate Hybrid & 10 & $\mathrm{R}$ \\
\hline 242 & BAH-4-A $\times$ K6-R SN:10-15 & TARI & Candidate Hybrid & 10 & $\mathrm{R}$ \\
\hline 243 & G5-K8 AD SN: $1 \times$ K7-R SN:6 & TARI & Candidate Hybrid & 10 & $\mathrm{R}$ \\
\hline 244 & BAH SN: $1(8525-A) \times K 7-R$ SN: 6 & TARI & Candidate Hybrid & 10 & $\mathrm{R}$ \\
\hline 245 & 0704-A $\times$ K7-R SN:2,3,4,5 & TARI & Candidate Hybrid & 10 & $R$ \\
\hline 247 & $62001-\mathrm{A} \times \mathrm{MMR}$ SN:8 & TARI & Candidate Hybrid & 10 & $\mathrm{R}$ \\
\hline 248 & 6626-A × K4-R SN:50 & TARI & Candidate Hybrid & 10 & $\mathrm{R}$ \\
\hline 249 & $6545 \times$ K6-R S.N:10-15 & TARI & Candidate Hybrid & 10 & $\mathrm{R}$ \\
\hline 250 & TQ97BR (TAT TOHUM) & TAT & Inbred Line & 10 & $\mathrm{R}$ \\
\hline 317 & F610730 & SOLTIS & Candidate Hybrid & 10 & $R$ \\
\hline 318 & F610731 & SOLTIS & Candidate Hybrid & 10 & $R$ \\
\hline 319 & F610732 & SOLTIS & Candidate Hybrid & 10 & $\mathrm{R}$ \\
\hline 388 & 6626-A × K4-R SN:58 & TARI & Candidate Hybrid & 10 & $\mathrm{R}$ \\
\hline 389 & 2517-A × K7-R SN:2,3,4,5 & TARI & Candidate Hybrid & 10 & $\mathrm{R}$ \\
\hline 390 & 2517-A × K6-R SN:10-15 (9786) & TARI & Candidate Hybrid & 10 & $R$ \\
\hline 391 & 2453-A × K4-R SN:46 & TARI & Candidate Hybrid & 10 & $R$ \\
\hline 393 & G5-K8 AD SN: $1 \times$ K6-R SN:10-15 & TARI & Candidate Hybrid & 10 & $\mathrm{R}$ \\
\hline 394 & G5-K8 AD SN:2 × K7-R SN:6 & TARI & Candidate Hybrid & 10 & $\mathrm{R}$ \\
\hline 395 & G5-K8 AD SN:3,4,5 × K7-R SN:6 & TARI & Candidate Hybrid & 10 & $\mathrm{R}$ \\
\hline 396 & G5-K8 AD SN:3,4,5 × K6-R SN:10-15 & TARI & Candidate Hybrid & 10 & $\mathrm{R}$ \\
\hline 397 & 8245-A × K7-R SN:6 & TARI & Candidate Hybrid & 10 & $R$ \\
\hline 398 & G4-K7 AD SN:4-9 × K7-R SN:6 & TARI & Candidate Hybrid & 10 & $R$ \\
\hline 400 & 2517-A × K7-R SN:6 (9702) & TARI & Candidate Hybrid & 10 & $R$ \\
\hline
\end{tabular}

R - resistant; T - tolerant; S - susceptible 
Table 11: Broomrape tests under conditions of natural infestation conducted in Malkara in 2008

\begin{tabular}{|c|c|c|c|c|c|}
\hline$\overline{\#}$ & Material & Origin & Type & Total plant & Result \\
\hline $\mathrm{C}$ & P-4223 (Resistant Check) & Pioneer & Commercial Hybrid & 9 & $R$ \\
\hline C & SANBRO (Susceptible Check) & Syngenta & Commercial Hybrid & 8 & $\mathrm{~S}$ \\
\hline 19 & $2453-A \times$ K4-R SN:117,118 & TARI & Candidate Hybrid & 5 & $\mathrm{R}$ \\
\hline 21 & $2453-A \times K 4-R$ SN:28-34 & TARI & Candidate Hybrid & 5 & $\mathrm{R}$ \\
\hline 22 & $2517-A \times$ K4-R SN:49,50,51,52 & TARI & Candidate Hybrid & 5 & $\mathrm{R}$ \\
\hline 24 & 2517-A × K4-R SN:182 & TARI & Candidate Hybrid & 4 & $\mathrm{R}$ \\
\hline 108 & $\mathrm{H}-114 / \mathrm{H}-115 \times \mathrm{K} 4-\mathrm{R}$ SN: 28,29-34 & TARI & Candidate Hybrid & 5 & $\mathrm{R}$ \\
\hline 114 & $\mathrm{H}-114 / \mathrm{H}-115 \times \mathrm{K} 4-\mathrm{R}$ SN: $119-129$ & TARI & Candidate Hybrid & 5 & $\mathrm{R}$ \\
\hline 125 & $\mathrm{H}-144 / \mathrm{H}-145 \times \mathrm{K} 4-\mathrm{R}$ SN: 28,29-34 & TARI & Candidate Hybrid & 5 & $\mathrm{R}$ \\
\hline 126 & $\mathrm{H}-144 / \mathrm{H}-145 \times \mathrm{K} 4-\mathrm{R}$ SN: $107-110$ & TARI & Candidate Hybrid & 5 & $\mathrm{R}$ \\
\hline 127 & $\mathrm{H}-144 / \mathrm{H}-145 \times \mathrm{K} 4-\mathrm{R} \mathrm{SN}: 119-129$ & TARI & Candidate Hybrid & 5 & $\mathrm{R}$ \\
\hline 131 & TF- 539-1/06 × K4-R SN: 28,29-34 & TARI & Candidate Hybrid & 7 & $\mathrm{R}$ \\
\hline 132 & TF- 539-1/06 × K4-R SN: 119,120-129 & TARI & Candidate Hybrid & 8 & $\mathrm{~T}$ \\
\hline 143 & TA-4142 / 06 × K4-R SN: 28,29-34 & TARI & Candidate Hybrid & 7 & $\mathrm{R}$ \\
\hline 144 & TA-4142 / 06 × K4-R SN: 130-133 & TARI & Candidate Hybrid & 7 & $\mathrm{~T}$ \\
\hline 151 & TH-2930 / 04 × K4-R SN: 28,29-34 & TARI & Candidate Hybrid & 7 & $\mathrm{R}$ \\
\hline 156 & TH-331-332 / 06 × K8-R SN: 10-13 & TARI & Candidate Hybrid & 7 & $\mathrm{R}$ \\
\hline 157 & TH-331-332 / 06 × K4-R SN: 28,29-34 & TARI & Candidate Hybrid & 8 & $\mathrm{R}$ \\
\hline 225 & $6388-A \times$ K4-R SN: 54,-59 & TARI & Candidate Hybrid & 6 & $\mathrm{R}$ \\
\hline 226 & $6388-\mathrm{A} \times \mathrm{K} 8-\mathrm{R}$ SN: 8,9 & TARI & Candidate Hybrid & 7 & $\mathrm{R}$ \\
\hline 229 & 7751-A × K4-R SN: 54-59 & TARI & Candidate Hybrid & 7 & $\mathrm{R}$ \\
\hline 240 & $2453-A \times$ K5-R SN:114,115,116-124 & TARI & Candidate Hybrid & 7 & $\mathrm{R}$ \\
\hline 241 & 0704-A × K4-R SN:130,131,132,133 & TARI & Candidate Hybrid & 7 & $\mathrm{~T}$ \\
\hline 242 & $6626-\mathrm{A} \times \mathrm{K} 4-\mathrm{R}$ SN: $64,65, \ldots . .73$ & TARI & Candidate Hybrid & 4 & $\mathrm{R}$ \\
\hline 243 & $6626-\mathrm{A} \times \mathrm{K} 4-\mathrm{R}$ SN:86,87 ...91 & TARI & Candidate Hybrid & 4 & $\mathrm{R}$ \\
\hline 244 & $6626-\mathrm{A} \times \mathrm{K} 4-\mathrm{R}$ SN:168,169 ..178 & TARI & Candidate Hybrid & 4 & $\mathrm{R}$ \\
\hline 245 & $2453-A \times K 5-R ~ S N: 125,126,127,128$ & TARI & Candidate Hybrid & 5 & $\mathrm{R}$ \\
\hline 246 & 2453-A × K5-R SN:83,84,85,86 & TARI & Candidate Hybrid & 4 & $\mathrm{R}$ \\
\hline 247 & $2453-\mathrm{A} \times \mathrm{K} 5-\mathrm{R}$ SN:67,68,69,70,71 & TARI & Candidate Hybrid & 5 & $\mathrm{R}$ \\
\hline 249 & $30002-A \times K 4-R$ SN:54,55...59 & TARI & Candidate Hybrid & 7 & $\mathrm{R}$ \\
\hline 266 & $2453-\mathrm{A} \times \mathrm{K} 8-\mathrm{R}$ SN: 17,18 & TARI & Candidate Hybrid & 7 & $\mathrm{R}$ \\
\hline 267 & $2453-A \times$ K5-R SN:139,140, ...145 & TARI & Candidate Hybrid & 7 & $\mathrm{R}$ \\
\hline 273 & $2453-\mathrm{A} \times \mathrm{K} 5-\mathrm{R}$ SN:57 ...66 & TARI & Candidate Hybrid & 7 & $\mathrm{~T}$ \\
\hline 274 & $2453-\mathrm{A} \times \mathrm{K} 5-\mathrm{R}$ SN:72...76 & TARI & Candidate Hybrid & 7 & $\mathrm{~T}$ \\
\hline 275 & $2453-A \times K 5-R$ SN:87,88 & TARI & Candidate Hybrid & 7 & $\mathrm{R}$ \\
\hline 278 & $2453-A \times$ K5-R SN:129...132 & TARI & Candidate Hybrid & 7 & $\mathrm{R}$ \\
\hline 281 & $2453-A \times K 5-R$ SN:168_..178 & TARI & Candidate Hybrid & 7 & $\mathrm{R}$ \\
\hline 284 & 2453-A × K5-R SN:12...15 & TARI & Candidate Hybrid & 7 & $\mathrm{R}$ \\
\hline 289 & $2453-\mathrm{A} \times \mathrm{K} 5-\mathrm{R}$ SN:14 ...16 & TARI & Candidate Hybrid & 7 & $\mathrm{~T}$ \\
\hline 291 & 6626-A × K5-R-SNO-57...66 & TARI & Candidate Hybrid & 7 & $\mathrm{~T}$ \\
\hline 292 & $6626-\mathrm{A} \times \mathrm{K} 8-\mathrm{R}-\mathrm{SNO}-7 . . .9$ & TARI & Candidate Hybrid & 7 & $\mathrm{~T}$ \\
\hline 294 & 6626-A × K8-R-SNO-14...15 & TARI & Candidate Hybrid & 7 & $\mathrm{R}$ \\
\hline
\end{tabular}

R - resistant; T - tolerant; S - susceptible 
sistant or non IMI resistant hybrids in Trakya region $(75 \%$ of the total production area) because in that part of Turkey $90 \%$ of sunflower acreage are infested with new broomrape races. Based on these results, TARI developed new resistant inbred lines and hybrids which will be released in 2009 and, after seed multiplication, these hybrids will appear on the market in 2010, for linoleic types. Oleic type broomrape- resistant hybrids will also be released in 2010 .

\section{REFERENCES}

Akhtouch, B., Munoz-Ruz, J., Melero-Vara, J.M, Fernández-Martínez, J.M. and Domínguez, J. 2002. Inheritance of resistance to race $\mathrm{F}$ of broomrape in sunflower lines of different origins. Plant Breeding 121: 266-268.

Fernández-Martínez, J.M., Melero-Vara, J.M, Mu oz-Ruz, J., Ruso, J. and Domínguez, J., 2000. Selection of wild and cultivated sunflower for resistance to a new broomrape race that overcomes resistance of the $\mathrm{Or}_{5}$ gene. Crop Science 40: 550-555.

Jan, C.C., Fernández-Martínez, J.M, Ruso, J. and Mu oz-Ruz, J., 2002. Registration of four sunflower germplasms with resistance to Orobanche cumana race F. Crop Science 42 : 2217-2218.

Kaya, Y., Demerci, M., Evci, G., 2004a. Sunflower (Helianthus annuus L.) breeding in Turkey for broomrape (Orobanche cernua Loeffl.) and herbicide resistance. Helia 27(40): 199210.

Kaya, Y., Evci, G., Pekcan, V. and Gucer, T., 2004b. Determining new broomrape infested areas, resistant lines and hybrids in Trakya Region of Turkey. Helia 27(40): 211-218.

Melero-Vara, J.M., Domínguez, J. and Fernández-Martínez, J.M., 2000. Update on sunflower broomrape situation in Spain: Racial status and sunflower breeding for resistance. Helia 23: 45-56.

Pérez-Vich, B., Akhtouch, B., Mu oz-Ruz, J., Fernández-Martínez, J.M. and Jan, C.C., 2002. Inheritance of resistance to the highly virulent race "F" of Orobanche cumana Wallr. in a sunflower line derived from interspecific amphiploids. Helia 25: 137-144.

Shindrova, P., 2006. Broomrape (Orobanche cumana Wallr.) in Bulgaria - distribution and race composition. Helia 29(44): 111-120.

Sukno, S., Melero-Vara, J.M. and Fernández-Martínez, J.M., 1999. Inheritance of resistance to Orobanche cernua Loelf. in six sunflower lines. Crop Science. 39: 674-678.

Tang, S., Heesacher, A., Kishore, V.K., Fernández, A., Sadik, E.S., Cole, G. and Knapp, S.J., 2003. Genetic mapping of the $\mathrm{Or}_{5}$ gene for resistance to Orobanche race E in sunflower. Crop Science 43: 1021-1028.

Vrânceanu, A.V., Tudor, V.A., Stoenescu, F.M. and Pirvu, N., 1980. Virulence groups of Orobanche cumana Wallr., differential hosts and resistance source genes in sunflower. Proc. $9^{\text {th }}$ Int. Sunflower Conf., 8-9 June. Torremolinos, Spain pp. 74-82.

\section{EVALUACIÓN DE LA RESISTENCIA DEL JOPO EN HÍBRIDOS DE GIRASOL}

\section{RESUMEN}

El jopo (Orobanche cernua Loeffl.) es el mayor problema que enfrenta el girasol en Turquía, en algunos países de Europa del este y en España. La investigación que abarca las pruebas de híbridos frente a este parásito en ensayos del Proyecto Nacional de Investigación de Girasol se llevó a cabo en Trakya, región que generó el $75 \%$ de la producción turca de girasol durante 2006-2008. Bajo infección natural, se probaron 306 materiales genéticos (híbridos comerciales y candidatos, líneas puras, etc.) en 2006, 443 en 2007 y 533 en 2008. De estos materiales, 12 de ellos se detectaron resistentes en 2006, 56 en 2007 y 140 en 2008. Durante el invierno, se realizaron inocula- 
ciones asistidas en cámara de crecimiento y en macetas. Semillas de jopo, colectadas en el área infectada naturalmente en Trakya, se utilizaron para determinar el comportamiento de híbridos y líneas frente a estas razas. En este estudio, 1752 materiales genéticos se probaron en 2006, 816 en 2007 y 1389 en 2008. De acuerdo a estas pruebas, 332 materiales genéticos fueron encontrados como resistentes a nuevas razas de jopo durante 2006, 94 en 2007 y 404 durante 2008.

\title{
EVALUATION DE LA RÉSISTANCE À l'Orobanche CHEZ LES HYBRIDES DE TOURNESOL
}

\author{
RESUME
}

L'Orobanche (Orobanche cernua Loeffl.) est l'un des problèmes majeurs pour la production de tournesol en Turquie, en Europe de l'Est et en Espagne. Des essais sur la tolérance à l'Orobanche des hybrides de tournesol ont été effectués, dans le cadre du National Sunflower Research Project, dans la région de Trakya, représentant $75 \%$ de la production de tournesol en Turquie en 2006, 2007 et 2008.

Ainsi, en condition d'infestation naturelle 306 cultivars de tournesol (hybrides commerciaux et en cours d'inscription, lignées parentales) ont été testés en 2006, 443 en 2007 et 533 en 2008. Parmi ce matériel, 12 cultivars ont été reconnus comme résistants à l'Orobanche en 2006, 56 en 2007 et 140 en 2008.

Durant l'hiver, les tests se sont poursuivis en serre et phytotrons sous infestation artificielle. Les semences d'Orobanche ont été prélevées dans les zones naturellement atteintes de Trakya et utilisées pour déterminer les réactions des hybrides et lignées développés vis-à-vis des différentes races.

Pour cette étude, 1752 cultivars ont été examinés en 2006, 816 en 2007 et 1389 en 2008. Les résultats montrent que 332 en 2006, 94 en 2007 et 404 en 2008 étaient résistants aux nouvelles races d'Orobanche. 
